\section{FLOW CYTOMETRY AND NANOSTRING PROVIDE A COMPREHENSIVE CELL- AND GENE-BASED TUMOR PROFILE FOLLOWING CHECKPOINT INHIBITION IN A MURINE BLADDER CANCER MODEL}

David Draper*, Philip Lapinski, Scott Wise. Labcorp Drug Development, Ann Arbor, MI, USA

Background Bladder cancer (BC) is the thirteenth leading cause of cancer-related deaths. ${ }^{1}$ Five checkpoint immunotherapies that target the PD-1/PD-L1 axis are FDA approved, and gene- and protein-based approaches are helping to identify new combination treatment strategies for therapeutic intervention. ${ }^{2}$ Using the murine MB49 model for BC, we demonstrate how non-targeted immune gene expression profiling can combine with flow cytometry to provide a gene and cell-specific signature for the tumor microenvironment and help identify potential targets for novel treatment approaches.

Methods Animals with established MB49 tumors were treated with anti-mPD-1 or isotype control antibodies. Tumors were collected 7 days after the last treatment. Flow cytometry examined anti-mPD-1 treatment-induced immunophenotypic modulation for eleven tumor-infiltrating immune subsets. The mouse PanCancer IO $360^{\mathrm{TM}}$ panel (NanoString) provided transcriptomic analysis of 770 immuno-oncology-related genes. The ROSALIND ${ }^{\mathrm{TM}}$ platform (OnRamp BioInformatics) was used to identify differentially regulated genes between treatment groups ( \pm 1.5 fold-change; $\mathrm{p}<0.05$ ).

Results Anti-mPD-1 had moderate anti-tumor activity, with a $58 \%$ tumor growth inhibition at day 18 post-implant in treated compared to control animals. Flow cytometry revealed anti-mPD-1 triggered an increase in tumor-infiltrating CD8+ $\mathrm{T}$ cells $(45 \%)$ compared to control animals. Furthermore, the CD8 + T cell phenotype was altered by anti-mPD-1 treatment. The percentage of CD8 $+\mathrm{T}$ cells that expressed ICOS and LAG-3 was increased in tumors from anti-mPD-1 treated animals $(22 \%$ and $35 \%$ respectively). A reduction in PD-1 expression was also observed (33\%). In myeloid cells, iNOS expression increased in tumor-associated macrophages from treated animals compared to controls. NanoString analysis revealed 62 genes were differentially regulated in tumors from anti-mPD-1 treated animals compared to controls. ROSALIND analysis classified 30 of the genes as regulators of interferon, cytotoxicity, antigen presentation, and cytokine/chemokine signaling. Also, among the genes upregulated by anti-mPD-1 were IDO, HAVCR2 (TIM-3), and CSFR1, which can promote tumor growth and are clinical targets actively being investigated for new immunotherapies.

Conclusions NanoString analysis complemented flow cytometry to provide a comprehensive profile of the MB49 tumor. Together, these data demonstrate that anti-mPD1 increases $\mathrm{T}$ cell recruitment into the tumor and upregulates the expression of genes known to enhance $\mathrm{T}$ cell recruitment and anti-tumor activity. iNOS protein upregulation indicates that anti-mPD-1 treatment may also exert effects by reprogramming M2 macrophages towards an M1 phenotype. Upregulation of IDO, HAVCR2, and CSFR1 genes may effectively counteract antimPD-1 treatment. Further investigation may elucidate clinical implications for inhibitors of these gene products as combination treatment partners with anti-mPD-1.

\section{REFERENCES}

1. Saginala K, Barsouk A, Aluru JS, Rawla P, Padala SA, Barsouk A. Epidemiology of bladder cancer. Med Sci 2020;1:15-26.
2. Lopez-Beltran A, Cimadamore A, Blanca A, Massari F, Vau N, Scarpelli M, Cheng L, Montironi R. Immune checkpoint inhibitors for the treatment of bladder cancer. Cancers 2021;1:131-146.

\section{Ethics Approval N/A}

http://dx.doi.org/10.1136/jitc-2021-SITC2021.275 